This is an electronic reprint of the original article. This reprint may differ from the original in pagination and typographic detail.

Author(s): Tarvainen, Olli; Angot, Julien; Izotov, Ivan; Skalyga, Vadim; Koivisto, Hannu; Thuillier, Thomas; Kalvas, Taneli; Lamy, Thierry

Title: $\quad$ Plasma instabilities of a charge breeder ECRIS

Year: $\quad 2017$

Version:

Please cite the original version:

Tarvainen, O., Angot, J., Izotov, I., Skalyga, V., Koivisto, H., Thuillier, T., Kalvas, T., \& Lamy, T. (2017). Plasma instabilities of a charge breeder ECRIS. Plasma Sources Science and Technology, 26(10), Article 105002. https://doi.org/10.1088/1361$6595 /$ aa8975

All material supplied via JYX is protected by copyright and other intellectual property rights, and duplication or sale of all or part of any of the repository collections is not permitted, except that material may be duplicated by you for your research use or educational purposes in electronic or print form. You must obtain permission for any other use. Electronic or print copies may not be offered, whether for sale or otherwise to anyone who is not an authorised user. 


\section{Plasma instabilities of a charge breeder ECRIS}

This content has been downloaded from IOPscience. Please scroll down to see the full text.

Download details:

IP Address: 130.234.75.187

This content was downloaded on 01/09/2017 at 07:43

Manuscript version: Accepted Manuscript

Tarvainen et al

To cite this article before publication: Tarvainen et al, 2017, Plasma Sources Sci. Technol., at press: https://doi.org/10.1088/1361-6595/aa8975

This Accepted Manuscript is: (c) 2017 IOP Publishing Ltd

During the embargo period (the 12 month period from the publication of the Version of Record of this article), the Accepted Manuscript is fully protected by copyright and cannot be reused or reposted elsewhere.

As the Version of Record of this article is going to be / has been published on a subscription basis, this Accepted Manuscript is available for reuse under a CC BY-NC-ND 3.0 licence after the 12 month embargo period.

After the embargo period, everyone is permitted to copy and redistribute this article for non-commercial purposes only, provided that they adhere to all the terms of the licence https://creativecommons.org/licences/by-nc-nd/3.0

Although reasonable endeavours have been taken to obtain all necessary permissions from third parties to include their copyrighted content within this article, their full citation and copyright line may not be present in this Accepted Manuscript version. Before using any content from this article, please refer to the Version of Record on IOPscience once published for full citation and copyright details, as permission will likely be required. All third party content is fully copyright protected, unless specifically stated otherwise in the figure caption in the Version of Record.

When available, you can view the Version of Record for this article at: http://iopscience.iop.org/article/10.1088/1361-6595/aa8975 


\title{
Plasma instabilities of a charge breeder ECRIS
}

\section{O Tarvainen ${ }^{1}$, J Angot ${ }^{2}$, I Izotov ${ }^{3}$, V Skalyga ${ }^{3}$, H Koivisto ${ }^{1}$, T} Thuillier $^{2}$, T Kalvas ${ }^{1}$ and T Lamy ${ }^{2}$

${ }^{1}$ University of Jyväskylä, Department of Physics, 40500 Jyväskylä, Finland

${ }^{2}$ Université Grenoble-Alpes, CNRS, IN2P3-LPSC, 53 avenue des martyrs 38026

Grenoble Cedex, France

${ }^{3}$ Institute of Applied Physics, RAS, 46 Ul'yanova St., 603950 Nizhny Novgorod, Russian Federation

E-mail: olli.tarvainen@jyu.fi

\begin{abstract}
Experimental observation of plasma instabilities in a charge breeder electron cyclotron resonance ion source (CB-ECRIS) is reported. It is demonstrated that the injection of ${ }^{133} \mathrm{Cs}^{+}$or ${ }^{85} \mathrm{Rb}^{+}$ion beam into the oxygen discharge of the CBECRIS can trigger electron cyclotron instabilities, which restricts the parameter space available for the optimization of the charge breeding efficiency. It is concluded that the transition from stable to unstable plasma regime is caused by gradual accumulation and ionization of $\mathrm{Cs} / \mathrm{Rb}$ and simultaneous change of the discharge parameters in 10 - $100 \mathrm{~ms}$ time scale, not by a prompt interaction between the incident ion beam and the ECRIS plasma. The instabilities lead to loss of ion confinement, which results to sputtering of the surfaces in contact with the plasma followed by up to an order of magnitude increase of impurity currents in the extracted $n+$ ion beam.
\end{abstract}




\section{Introduction}

Charge breeder electron cyclotron resonance ion sources (CB-ECRIS) [1] are used as injectors for post-acceleration of radioactive ion beams [2]. The charge breeding process in the ECRIS plasma involves multiple processes: thermalization of the injected $1+$ ions with the plasma ions in ion-ion collisions, subsequent multiplication of the charge state of the injected element by electron impact ionization, and extraction of the $n+$ ions, all occurring simultaneously in continuous operation of the ion source [3]. The primary benefits of ECRIS charge breeders in comparison to alternative techniques, especially electron beam ion sources (EBIS) [4], include the readiness for both continuous and pulsed operation and high capacity for the accumulation of the injected charge. On the other hand, ECRIS charge breeders have certain drawbacks including broad charge state distribution and pervasive impurity background of the extracted beam, which complicate the post-acceleration of radioactive isotopes especially in the case of linear accelerators, favoring the use of EBIS devices at low radioactive beam intensities below $10^{4}$ pps [5]. Nevertheless, ECRIS charge breeders have found their purpose in radioactive beam facilities in operation and under construction, e.g. ISAC at TRIUMF [6], SPIRAL1 / SPIRAL2 at GANIL [7, 8], SPES at INFN-LNL [9] and HIE-ISOLDE at CERN [10].

It has been shown recently that the parameter space available for the optimization of the performances of conventional ECR ion sources is restricted by kinetic instabilities [11]. Such instabilities, first reported for ECRIS in Ref. [12], arise from the resonant nature of the stochastic electron heating process resulting to anisotropic electron velocity distribution (EVD) consisting of several electron populations characterized by different average energies [13, 14]. The instabilities are driven by the hot electrons which play only a minor role in electron impact ionization [15] but could significantly contribute to the plasma confinement by creating an electrostatic potential dip in the core of the plasma [16] and, thus, prolonging the high charge state ion confinement times [17] in the absence of plasma instabilities. The threshold between quiescent and turbulent plasma regimes is affected by all the main tuning parameters of the ECRIS, i.e. magnetic field strength and topology (plasma dimensions, resonance gradient etc.), microwave power, neutral gas pressure and neutral gas species $[11,12,18]$. Magnetic field strength and the microwaye power are believed to affect the growth rate of the instability while the effects of the neutral gas pressure and composition can be explained by inelastic collisions affecting the damping rate of the instability [12].

The purpose of the research reported hereafter is to study the influence of $1+$ injection on the stability of the ECRIS plasma. This was accomplished by (i) studying the effect of kinetic plasma instabilities on the ECRIS charge breeding efficiency of cesium $\left({ }^{133} \mathrm{Cs}\right)$ and rubidium $\left({ }^{85} \mathrm{Rb}\right)$ ion beams, (ii) demonstrating that the interaction of the $1+$ beam with the charge breeder plasma can trigger instabilities, which underlines the potential of the $1+$ injection as a plasma diagnostics technique shedding light on the charge breeding process and (iii) showing that the occurrence of the instabilities affects the purity of the extracted ion beam as a result of plasma-wall interactions (desorption 
Plasma instabilities of a charge breeder ECRIS

and sputtering) increasing the concentration of impurities in the charge breeder ECRIS plasma.

\section{Experimental setup}

The experimental data were taken with the 14.5 GHz PHOENIX ECR charge breeder [19] at Laboratoire de Physique Subatomique et de Cosmologie (LPSC). All the experiments reported hereafter were conducted using oxygen as a buffer gas for the charge breeder discharge sustained by $14.52 \mathrm{GHz}$ microwave radiation. Cesium $\left({ }^{133} \mathrm{Cs}\right)$ and rubidium $\left({ }^{85} \mathrm{Rb}\right)$ were chosen as injected $1+$ ion species for the following reasons: (i) they are alkali metals condensing on the walls of the charge breeder plasma chamber, which simplifies the interpretation of the diagnostics signals (so-called $\Delta V$-curves) related to the capture of the incident $1+$ beam in comparison to gaseous elements [20], (ii) they are significantly heavier than the charge breeder buffer gas (oxygen) ions, which favors the kinematics of the capture process by ion-ion collisions [3, 21], (iii) their atomic numbers ( $Z=55$ and $Z=37$ for $\mathrm{Cs}$ and Rb, respectively) are sufficiently high to affect the properties of the charge breeder oxygen $(Z=8)$ plasma through accumulation and ionization and (iv) they have been used extensively in the past for diagnostics [22] and efficiency studies $[23,24]$ of the given charge breeder ECRIS, which helps placing the research in appropriate context.

The LPSC charge breeder test stand shown in Fig. 1 has been thoroughly described elsewhere [25] and only its main features are summarized below. The ${ }^{133} \mathrm{Cs}^{1+}$ and ${ }^{85} \mathrm{Rb}^{1+}$ ion beams are produced with a thermionic ion gun manufactured by Heat Wave Labs. The intensity of the $1+$ ion beam is adjusted by controlling the temperature of the porous ionizer body into which the alkali metal has been fused. The $1+$ beams are $\mathrm{m} / q$-analyzed with a 90 degree bending magnet and their currents are monitored with a Faraday cup placed downstream from the magnet. The $1+$ ion beam is pulsed by applying a $320 \mathrm{~V}$ voltage between the parallel plates (separated by $30 \mathrm{~mm}$ ) of an electrostatic steerer placed between the $1+$ ion source and the bending magnet. The 90 $\%$ rise time of the resulting transverse electric field deflecting the beam is $44 \mu \mathrm{s}$, which is negligible in comparison to charge breeding times discussed below. The energy of the $1+$ ions entering the charge breeder is controlled by the voltage difference $\Delta V$ applied between the $1+$ ion source and the charge breeder ECRIS. Due to the positive plasma potential of the ECR discharge, $\Delta V$ must be negative in order to prevent reflection of the incident $1+$ ions. In the case of alkali metals the optimum value of $\Delta V$ for maximizing the capture efficiency of the injected beam is in the range of $3-10 \mathrm{~V}$ (negative). In the ECRIS plasma the thermalized alkali ions undergo consecutive ionizing electron impact collisions resulting to a charge state distribution (CSD) characteristic of ECR ion sources. The charge bred ions are extracted from the ECRIS together with the buffer gas (oxygen) ions by the potential difference applied between the source and its puller electrode $(15 \mathrm{kV}$ in the experiments reported here) and $m / q$-analyzed with a 120 degree bending magnet. The charge breeding efficiencies were determined by comparing the $1+$ 
and $n+$ ion beam currents measured upstream from the ECRIS and from the Faraday cup at the end of the beam line, respectively. The $n+$ currents were also measured after switching off the $1+$ injection, thus allowing to subtract the contribution of the background current on the charge breeding efficiencies. The Faraday cup of the $n+$ beamline connected to a picoammeter was also used for monitoring the temporal stability of the beam currents extracted from the charge breeder. The horizontal Allison-type emittance scanner placed between the 120 degree bending magnet and the $n+$ Faraday cup was used for recording high resolution CSDs, which allowed monitoring the level of impurities within the extracted beam. The scanner is equipped with a pair of $0.2 \mathrm{~mm}$ entrance and exit slits, separated by a distance of $64 \mathrm{~mm}$, placed in front of the Faraday cup measuring the current, which improves the resolution of the CSD in comparison to the $n+$ Faraday cup.

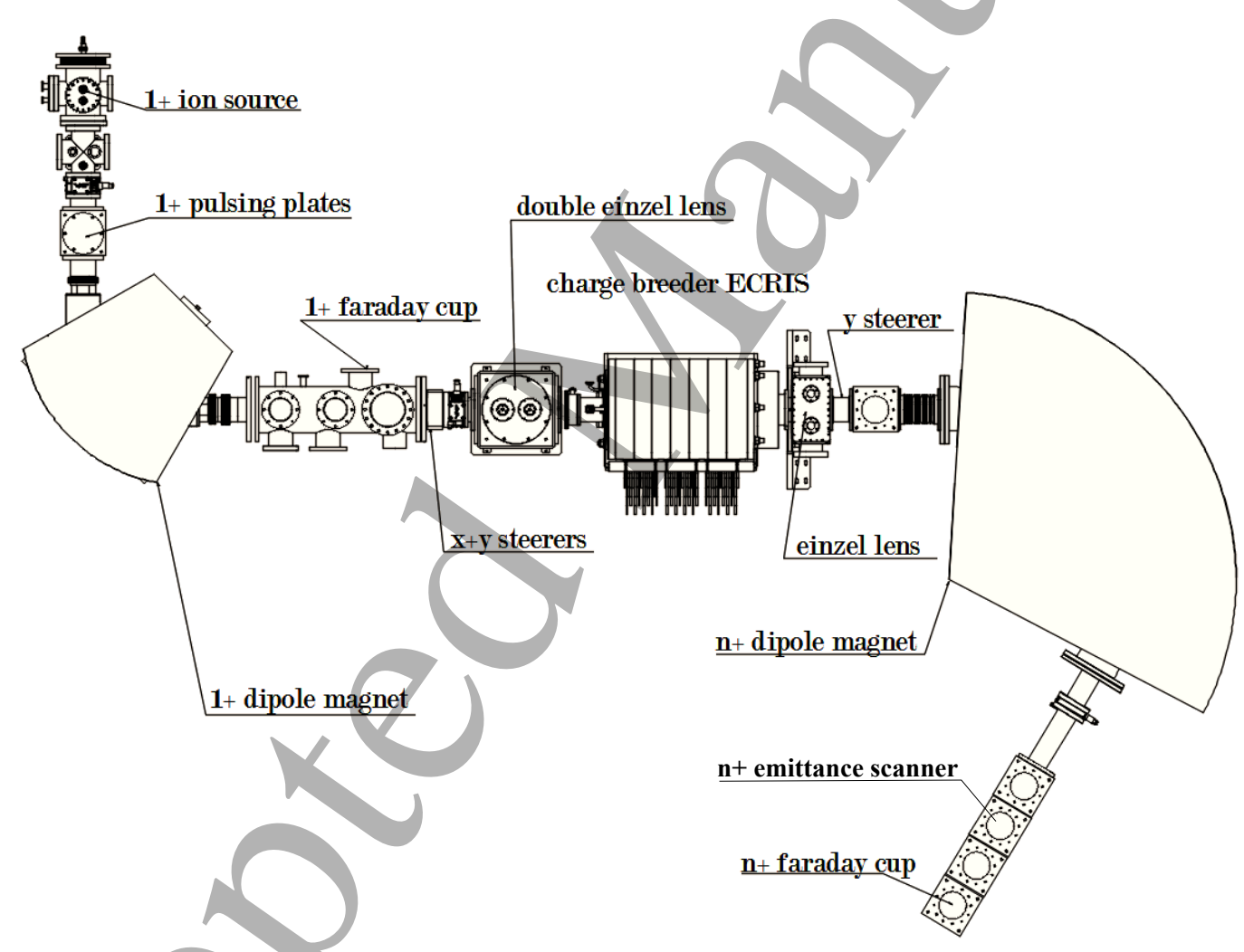

Figure 1. Layout of the charge breeder test bench.

The regular configuration of the charge breeder test stand was appended with diagnostics enabling to identify the existence of kinetic plasma instabilities. The diagnostics setup is presented schematically in Fig. 2. A low-barrier Schottky diode detector (Keysight, model 8473C), sensitive to microwave radiation in the frequency range of $10 \mathrm{MHz}-26.5 \mathrm{GHz}$, was connected to the secondary waveguide port (WR-62) of the charge breeder ECRIS. The microwave signal was guided into the detector through a high-voltage break, approximately $8 \mathrm{~m}$ of WR-62 waveguide, $13.2 \mathrm{GHz}$ low pass filter blocking the transmitted microwave signal of the klystron at $14.52 \mathrm{GHZ}$, WR-62 to WR-75 and WR75 to coaxial adapters, $1.5 \mathrm{~m}$ coaxial signal cable, $-70-0 \mathrm{~dB}$ adjustable 
attenuator (Keysight, model 8495D) and power limiter (Keysight, model N9355C). The microwave detector transforms the microwave radiation into a voltage signal and can be connected to an oscilloscope to monitor and record short bursts of microwave emission characteristic of electron cyclotron instabilities. The frequency range of the microwave bursts associated with the instabilities was probed by dividing the signal with a magic-tee and connecting different high-pass filters to one of its output ports. The signals recorded from the filtered and unfiltered ports were compared using two identical Schottky diodes, which enables narrowing the frequency range of the microwave emission as explained in detail in Ref. [26]. Another characteristic feature of the kinetic instabilities is the periodic fluctuation of the (thick-target) bremsstrahlung radiation. The bremsstrahlung bursts are generated by electrons expelled from the plasma by the instability. The bremsstrahlung signal was monitored with a detector, placed at a distance of about $0.5 \mathrm{~m}$ from the injection end of the charge breeder, consisting of a BGO (bismuth germanate) scintillator and a photomultiplier operated in current-mode i.e. with negative cathode voltage and the anode connected directly into a Keithley picoammeter. In such configuration the detector signal, is proportional to the energy content $n_{e}\left\langle E_{e}\right\rangle$ (or power flux $\frac{d\left(N_{e}\left\langle E_{e}\right\rangle\right)}{d t}$ ) of the hot electrons escaping the magnetically confined plasma and interacting with the plasma chamber walls. The temporal variation of the scintillator signal was monitored and recorded with an oscilloscope. Together with the $n+$ Faraday cup these diagnostics allowed detecting the instabilities and characterizing their effect on the performance of the charge breeder.

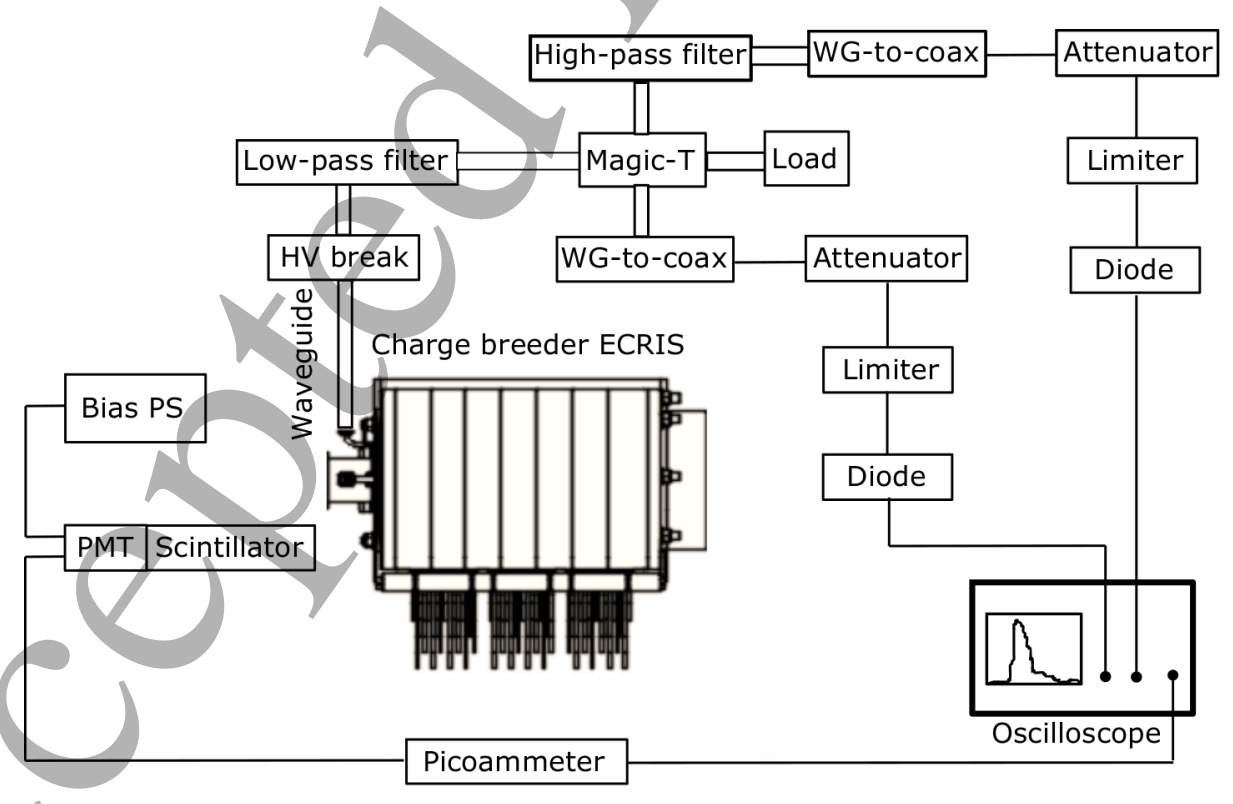

Figure 2. Schematic layout of the diagnostics setup. The main components are the diode detector(s) and scintillator probing the microwave and x-ray (bremsstrahlung) emissions, respectively. 


\section{Experimental results and discussion}

The experiments consisted of three stages; the characterization of the instabilities of the charge breeder plasma without $1+$ injection, the study of the influence of the ${ }^{133} \mathrm{Cs}^{1+}$ and ${ }^{85} \mathrm{Rb}^{1+}$ injection on the instability threshold, and the identification of the impact of the instabilities on the level of impurities in the extracted beam.

The transition threshold from stable to unstable regime is dictated by the magnetic field strength of the ECRIS. The magnetic field of the PHOENIX charge breeder is a superposition of a permanent magnet sextupole and solenoid field generated by three electromagnet coils. Although the effect of the magnetic field topology on the instability threshold was not studied exhaustively, it was observed that the plasma stability is sensitive to the current of the central coil, which primarily affects the magnetic field strength near the center of the trap, i.e. $B_{\text {min }}$, as well as the average gradient of the magnetic field on the resonance surface. Therefore in the experiments described hereafter, the $B_{\text {min }} / B_{E C R}$ ratio, used for characterizing the magnetic field strength in the following subsections, was varied in the range of $0.75-0.90$ by changing the current in the central coil only. The effect of the central coil current on the magnetic field is demonstrated in Fig. 3 showing the magnetic field profiles (simulated with 3D RADIA software [27]) on the source axis at two different currents of $271 \mathrm{~A}$ and $361 \mathrm{~A}$ while keeping the injection and extraction coil currents at $1123 \mathrm{~A}$ and $730 \mathrm{~A}$, respectively. These magnetic field configurations correspond to a $B_{\min } / B_{E C R^{-r a n g e}}$ of $0.78-0.88$ in which the transition from stable to unstable plassma occurs. The corresponding magnetic field (on-axis) gradients at the (non-relativistic electron) resonance on injection and extraction sides are also listed in the figure. It is worth noting that the on-axis field gradients of the $14.5 \mathrm{GHz}$ PHOENIX ECRIS are significantly lower than those reported for the transition from stable to unstable plasma in the case of the JYFL $14 \mathrm{GHz}$ ECRIS [11]. The main differences between the two ion sources are the injection and radial field strengths, i.e. $2 \mathrm{~T}$ vs. $1.2 \mathrm{~T}$ and $1.1 \mathrm{~T}$ vs. $0.8 \mathrm{~T}$ (sextupole only), respectively. Altogether this implies that due to its effect on electron confinement, the absolute field strength could play an important role in determining the transition from stable to unstable plasma/regime instead of the magnetic field gradient discussed in Ref. [11]. Thus, the role of the magnetic field strength and topology (gradient) should be studied in detail in order to resolve their respective effects on the instability threshold. This is further motivated by a recent investigation by Benitez et al. [28] showing that the spectral temperature of the bremsstrahlung in the energy range of 80-300 keV, which can be considered as an indicator of the average electron energy within the hot tail of the electron distribution in ECRIS plasmas, depends only on the absolute value of $B_{\min }$, not on the on-axis field gradient nor the plasma volume enclosed by the ECR zone.

Another parameter affecting the stability of the plasma was observed to be the applied microwave power which was adjusted in the range of 40-400 W (typical for the PHOENIX charge breeder ECRIS). The neutral gas feed rate was kept constant during each parametric sweep described in the following subsections. 


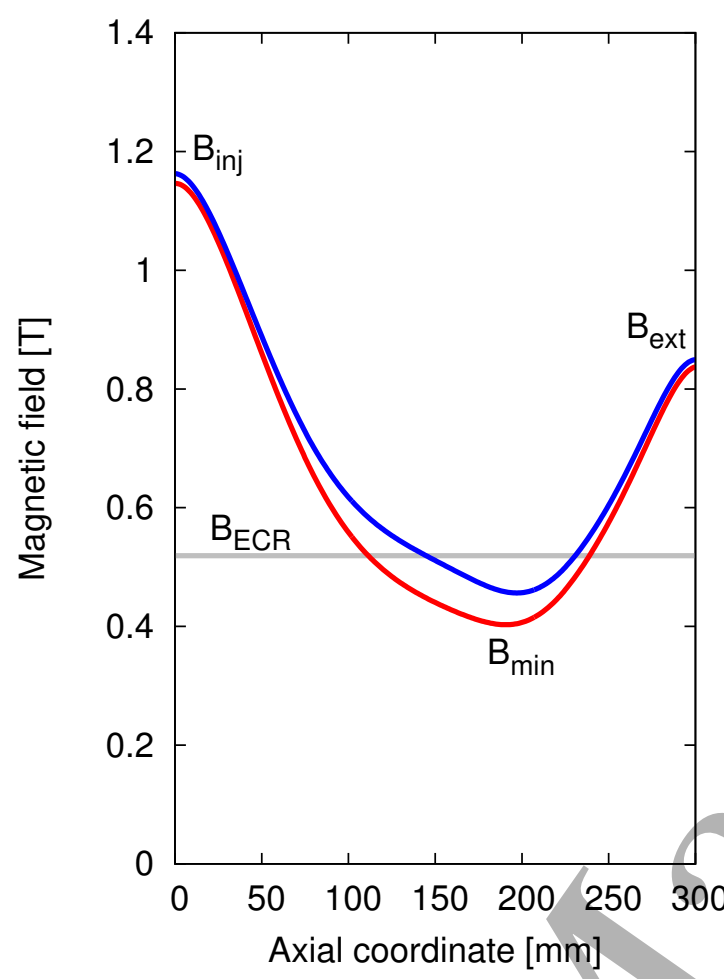

$$
\begin{aligned}
& \mathrm{B}_{\text {min }} / \mathrm{B}_{\mathrm{ECR}}=0.78 \\
& \nabla \mathrm{B}_{\text {inj }}=2.9 \mathrm{~T} / \mathrm{m} \\
& \nabla \mathrm{B}_{\text {ext }}=4.6 \mathrm{~T} / \mathrm{m}
\end{aligned}
$$

Stable

Figure 3. Axial magnetic field profiles corresponding to $B_{\min } / B_{E C R}$-values typical to stable (0.78) and unstable (0.88) oxygen plasmas of the $14.5 \mathrm{GHz}$ PHOENIX ECR charge breeder.

\subsection{Characterization of the plasma instabilities}

Typical diagnostics signals, characteristic to the cyclotron-type kinetic instabilities of the charge breeder ECRIS plasma, are displayed in Fig. 4. The figure shows normalized microwave emission and bremsstrahlung signals, as well as the temporal evolution of the extracted currents of oxygen charge states $\mathrm{O}^{+}, \mathrm{O}^{4+}$ and $\mathrm{O}^{7+}$, following an onset of the instability at $t=0$. The beam currents are normalized to their respective values at $t=0$, which enables emphasizing the difference between the response of low, medium and high charge state ions to the onset of the instability.

The first indication of the instability is an intense microwave burst lasting 100-200 ns, followed by an abrupt increase in bremsstrahlung emission for several hundreds of microseconds due to electrons driven into the loss cone by the amplified plasma wave. Furthermore, the instability causes an evident drop of the ion currents of all oxygen charge states, followed by a recovery phase. The initial drop of the beam current is caused by the loss of ion confinement and a mismatch of the ion optics at elevated plasma potential resulting to significant energy spread of the extracted beam [18]. During the recovery low, medium and high charge states exhibit different behaviors. Low charge state ion currents (e.g. $\mathrm{O}^{+}$) recover fast, reaching their maximum value overshooting the steady-state current by almost $20 \%$ approximately 5-10 ms after the instability. Such overshoot can be explained by an abrupt shift of the charge state distribution following 

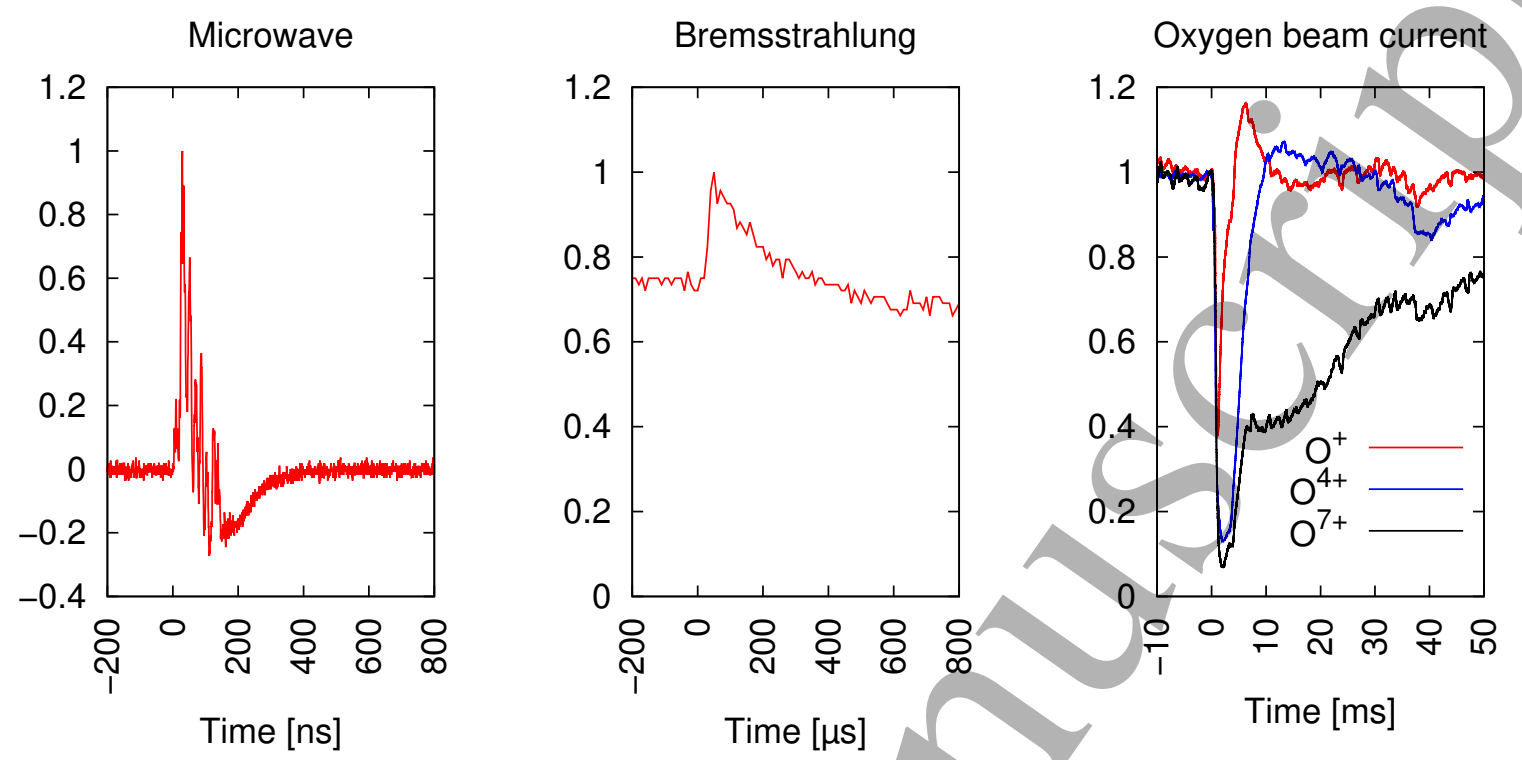

Figure 4. Typical (normalized) diagnostics signals related to electron cyclotron instabilities of the charge breeder ECRIS plasma: microwave emission (left), bremsstrahlung power flux (middle) and oxygen beam current variation of low $\left(\mathrm{O}^{+}\right)$, medium $\left(\mathrm{O}^{4+}\right)$ and high $\left(\mathrm{O}^{7+}\right)$ charge state (right).

the instability affected by the losses of high charge state ions and their neutralization on the chamber walls. Medium charge state ion beam currents (e.g. $\mathrm{O}^{4+}$ ) return back to their initial values typically in $10-20 \mathrm{~ms}$ while high charge state (e.g. $\mathrm{O}^{7+}$ ) beams exhibit a slow recovery of several tens of milliseconds, reflecting the time required for accumulation of plasma energy content and step-wise ionization of highly charged ions. The repetition rate of the instabilities is typically on the order of $10 \mathrm{~Hz}-1 \mathrm{kHz}$, which translates to 1-100 ms temporal interval between consecutive onsets. The given range of the repetition rate matches with the timescale for the multicharged oxygen plasma to reach its equilibrium charge state distribution. Such abrupt fluctuation of the buffer gas discharge can be expected to be detrimental for the charge breeding application because the breeding times (90\% rise times) of high charge state Cs ions, for example, are longer than $100 \mathrm{~ms}$ [29].

In order to complete the characterization of the instabilities occurring in the oxygen plasma, the frequency range of the microwave emission was estimated with a combination of waveguide filters forming a band pass system and, hence, restricting the frequencies of the detected signal either to $9.5-13.2 \mathrm{GHz}$ (the lower limit determined by the cut-off of the WR62 waveguide) or 11.8-13.2 GHz. Figure 5 shows examples of the recorded microwave emission signals of two instability events.

The microwave burst depicted on the left side subfigure is observed with both diodes (filters) which implies that the emission occurs in the range of 11.8-13.2 GHz. The subfigure on right shows a microwave burst consisting of two "emission packets", the first one emitting in the range of $11.8-13.2 \mathrm{GHz}$ and the second one in the range of 9.5- 


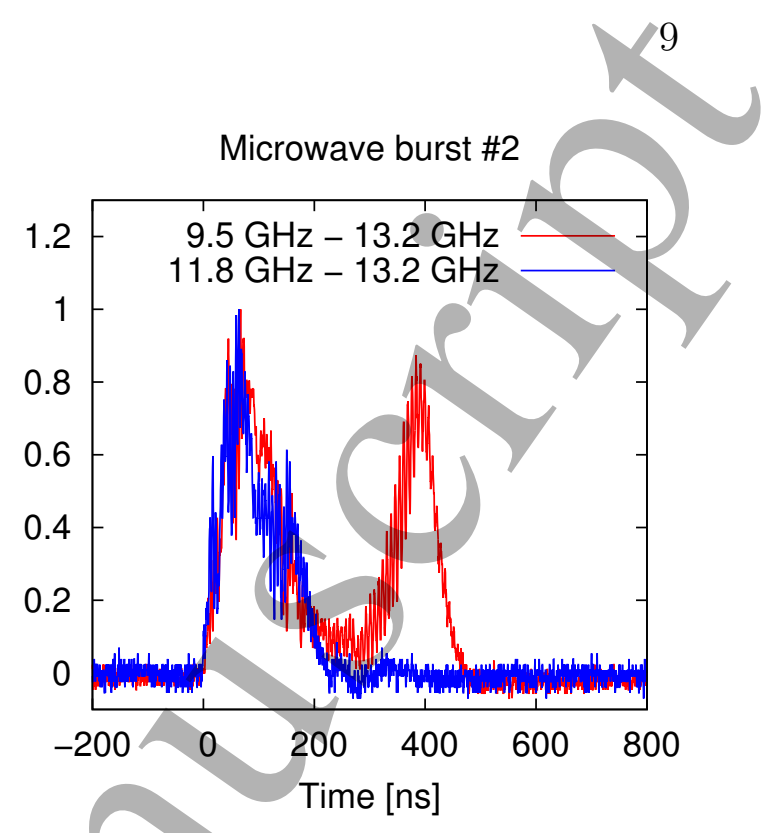

Figure 5. Normalized microwave emission signals of two oxygen plasma instability events measured through $9.5-13.2 \mathrm{GHz}$ and $11.8-13.2 \mathrm{GHz}$ band pass filters.

11.8 GHz. The falling tone of the microwave emission was found to be a characteristic feature of the instability events consisting of multiple "emission packets". This confirms an earlier observation [30] with the JYFL 14 GHz ECRIS and, therefore, suggests that the main band of the microwave emission being found at frequencies below the primary electron heating frequency and exhibiting a falling tone, are fundamental features of the electron cyclotron instabilities of ECRIS plasmas.

\subsection{The effect of ${ }^{133} C s$ and ${ }^{85}$ Rb injection on the plasma instabilities}

The effect of $1+$ beam injection on the transition from stable to unstable plasma and the subsequent impact on the charge breeding efficiency of alkali metal ions was studied using ${ }^{133} \mathrm{Cs}^{26+}$ and ${ }^{85} \mathrm{Rb}^{19+}$ as representative examples of the high charge state ion population. These ions were chosen since the breeding efficiencies of $\mathrm{Cs}$ and $\mathrm{Rb}$ in oxygen discharge typically reach their maxima near (or at) the given charge states as shown in Fig. 6.

It has been shown that the $1+$ injection and accumulation affects the charge state distribution of the buffer gas ions due to energy exchange in ion-ion collisions [31, 22], referred as gas mixing $[32,33]$ in plasmas of conventional ECR ion sources. In charge breeders the extracted current of high charge state buffer gas ions are affected more than the low charge states and the significance of the effect depends on the mass of the injected ions [22]. Recently, a particle-in-cell simulation code applied to ECRIS was used to propose a mechanism to explain how a small quantity of massive ions can significantly affect the charge state distribution of oxygen plasma [34]. The effect is demonstrated in Fig. 7 that shows the extracted currents (normalized) of $\mathrm{Cs}^{26+}$ and 


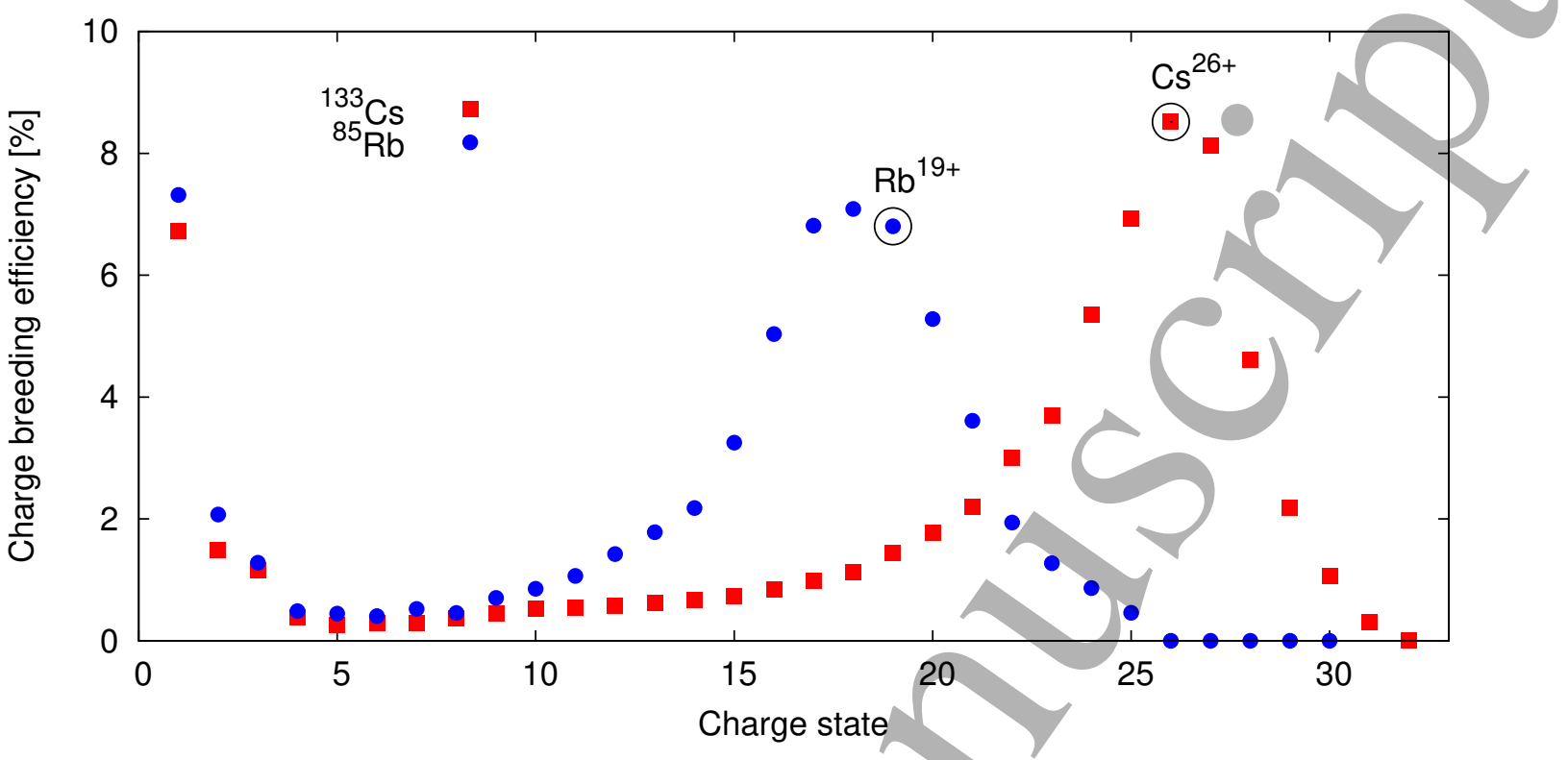

Figure 6. Typical distribution of charge breeding efficiencies of ${ }^{133} \mathrm{Cs}$ and ${ }^{85} \mathrm{Rb}$ ion beams in oxygen discharge of the PHOENIX charge breeder ECRIS.

$\mathrm{O}^{6+}$ ions as a function of the potential difference $(\Delta V)$ between the $1+$ ion source and the charge breeder ECRIS when $100 \mathrm{nA}$ of $\mathrm{Cs}^{+}$is injected (left). The given example was recorded with $310 \mathrm{~W}$ microwave power at $B_{\min } / B_{E C R}$-ratio of 0.84 . The figure also shows the extracted currents (normalized) of the afore-mentioned charge states as a function of time (right, $t=0$ corresponds to switching on the $1+$ injection). In this case the data was collected with an injection of $640 \mathrm{nA}$ of $\mathrm{Cs}^{+}, \Delta V=-6 \mathrm{~V}, 310 \mathrm{~W}$ microwave power and $B_{\min } / B_{E C R}$-ratio of 0.83 .

The figure summarizes several important features of the effect of $1+$ injection on the charge breeder plasma: (i) the significance of the effect, i.e. drop of high charge state oxygen beam current(s), depends on the injected current, (ii) the effect is caused by the capture of the injected $1+$ ions as demonstrated by the $\Delta V$-curves of $\mathrm{Cs}^{26+}$ and $\mathrm{O}^{6+}$ mirroring each other and (iii) the temporal behavior of the $\mathrm{O}^{6+}$ current is determined by the accumulation of $\mathrm{Cs}$, not by a prompt interaction of the injected beam and the buffer gas plasma, as demonstrated by the response time of several hundreds of milliseconds. Altogether this implies that $1+$ injection can drastically affect the properties of the buffer gas plasma, including the electron density and energy distribution, through gradual accumulation and ionization of the injected ion species. This is highlighted in Fig. 8 showing the extracted beam current of $\mathrm{Cs}^{26+}$ and the bremsstrahlung power flux $(\mathrm{BGO}+\mathrm{PMT}$ detector signal) as a function of $\Delta V$ under the presence of the plasma instability triggered by the injection of $200 \mathrm{nA}$ of $\mathrm{Cs}^{+}$beam into the oxygen discharge of the charge breeder. The corresponding ion source settings are $310 \mathrm{~W}$ microwave power and $B_{\min } / B_{E C R^{-r a t i o}}$ of 0.84 . The data are normalized with respect to the maximum signal recorded at stable source settings.

In the $-3.5 \mathrm{~V}<\Delta V<0 \mathrm{~V}$ range the incident $1+$ ions are reflected back by 

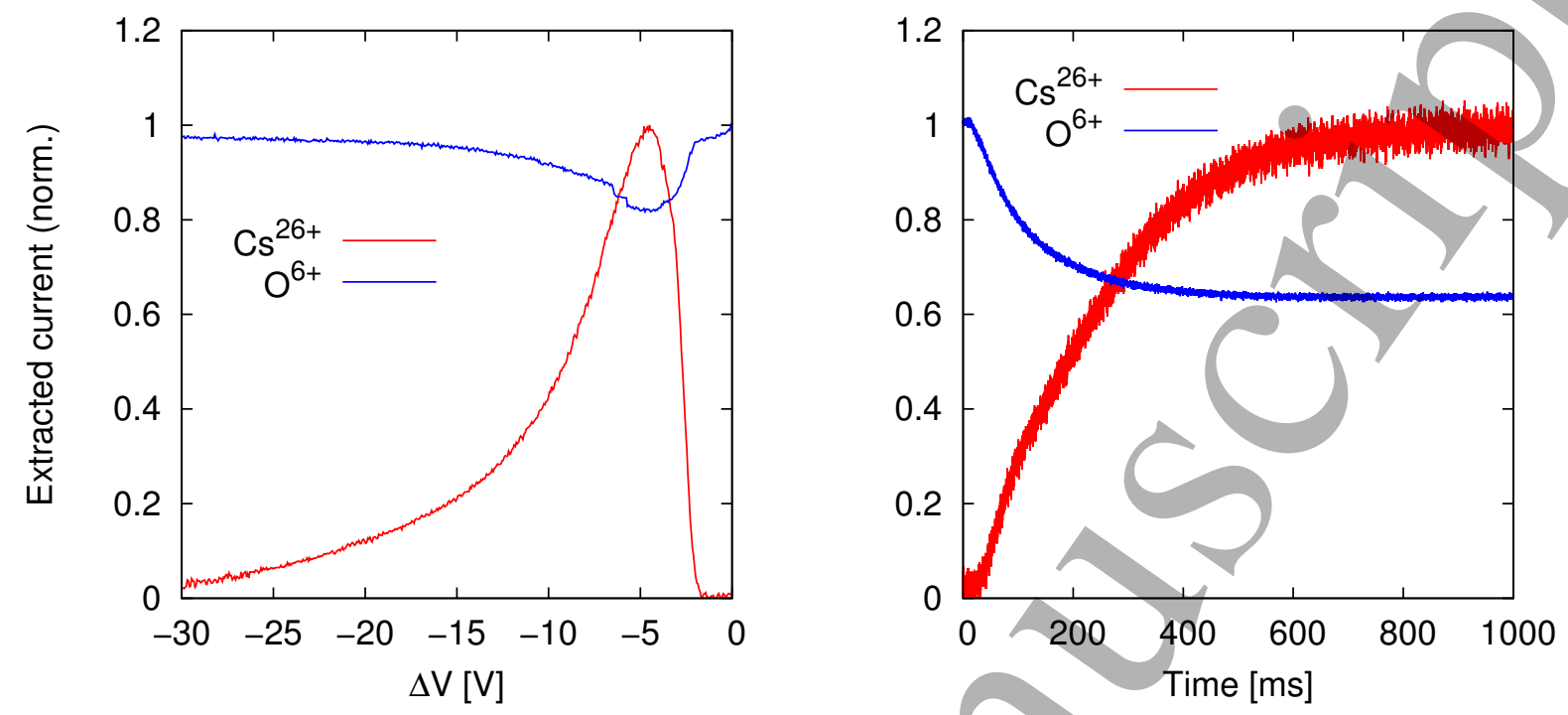

Figure 7. Normalized extracted currents of $\mathrm{Cs}^{26+}$ and $\mathrm{O}^{6+}$ as a function of $\Delta V$ (left) and their temporal response to $\mathrm{Cs}^{+}$injection (right). The injected $1+$ currents are 100 $\mathrm{nA}$ and $640 \mathrm{nA}$, respectively.

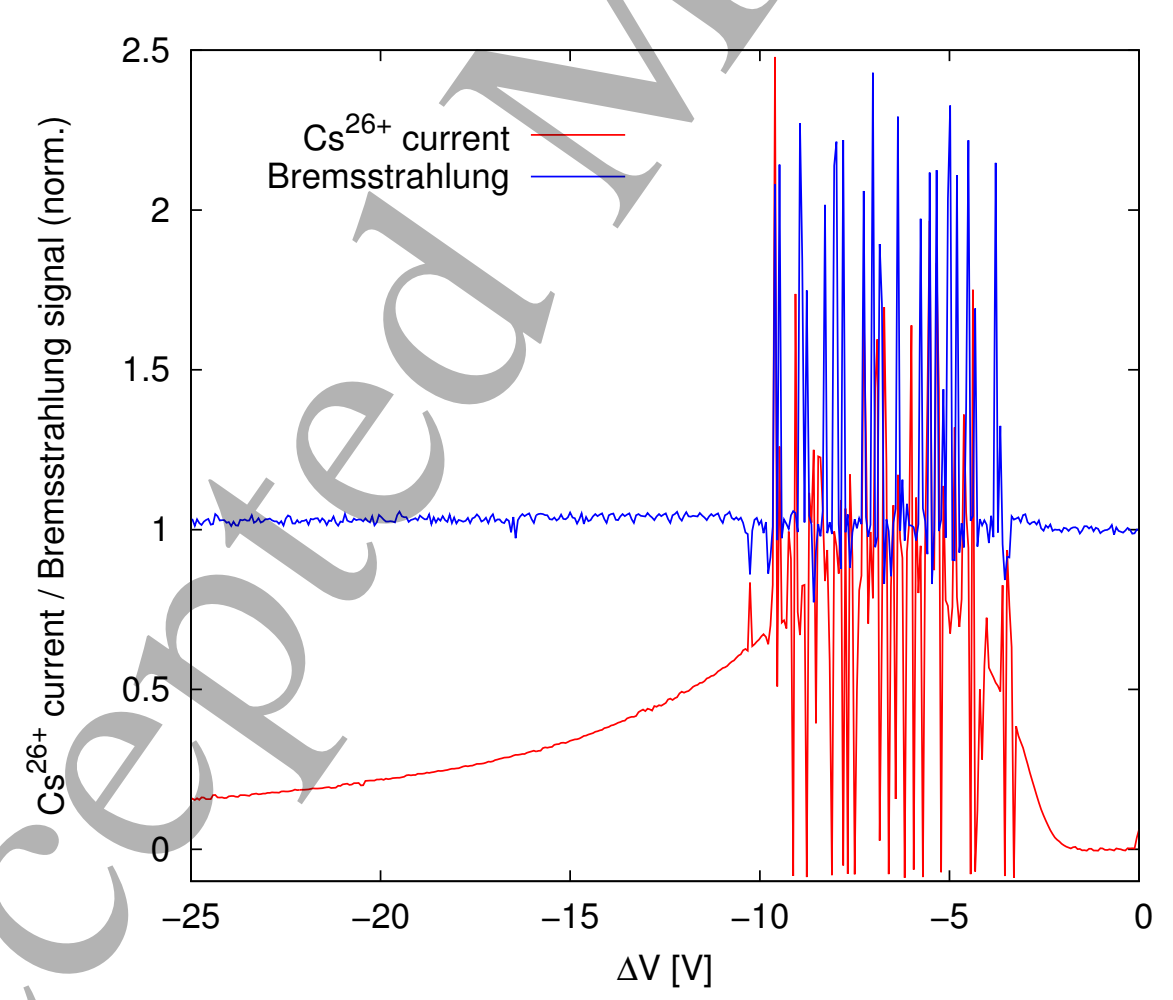

Figure 8. The extracted beam current of $\mathrm{Cs}^{26+}$ and the bremsstrahlung power flux as a function of $\Delta V$ under the presence of plasma instability triggered by the injection of $200 \mathrm{nA}$ of $\mathrm{Cs}^{+}$beam into the oxygen discharge of the charge breeder. 
the plasma potential of the charge breeder and therefore do not significantly affect the properties of the oxygen discharge. On the contrary, when $-10.5 \mathrm{~V}<\Delta V<-3.5 \mathrm{~V}$ a significant fraction of the $1+$ ions is captured as indicated by the increase of the high charge state Cs current. In the given range severe instabilities exist as demonstrated by the extracted $\mathrm{Cs}^{26+}$ beam current and bremsstrahlung signals exhibiting strong fluctuations. The instabilities are caused by the change of the plasma properties induced by the capture of the $1+$ ions. At $\Delta V<-10.5 \mathrm{~V}$ the energy of the injected $1+$ ions exceeds the optimum for their thermalization and they can propagate through the oxygen plasma as demonstrated both experimentally [22, 35] and numerically [36]. The reduced level of interaction with the oxygen ions causes the instabilities to disappear at high $1+$ energies. Simultaneous measurement of the beam current and bremsstrahlung power flux fluctuations confirms that the observed behavior can be attributed to kinetic plasma instabilities. This underlines the fact that the capture and accumulation of the injected $1+$ ions affects the electron velocity distribution of the charge breeder plasma.

In order to probe whether the plasma instability is caused by a prompt interaction of the $1+$ beam with the background plasma [37], as discussed in the specific case of an ECRIS charge breeder in Ref. [36], or by gradual accumulation of the injected species and subsequent modification of the plasma parameters, the $\mathrm{Cs}^{+}$beam was pulsed and the delay between the leading edge of Cs injection and the first appearance of the instabilities was measured at injected currents in the range of 90-1020 nA with $\Delta V=-5$ V. The result is shown in Fig. 9(a). The vertical error bar represents $8 \%$ statistical variation of the delay determined at injected currents of 200 and $915 \mathrm{nA}$. The experiment was realized by first setting the injected $1+$ current to $1020 \mathrm{nA}$ and then reducing the current of the $1+$ ion source heater which results to decreasing $1+$ output. As the $1+$ current was slowly decreasing, the afore-mentioned delay was recorded. Thus, the horizontal error bar corresponds to the estimated decay of the $1+$ beam current during the acquisition of each data point. The microwave power and magnetic field settings were $300 \mathrm{~W}$ and $B_{\min } / B_{E C R}=0.82$. The figure also shows the best fit to the experimental data calculated with Gnuplot (version 5.0). Alternatively, figure 9(b) shows the (total) injected charge required to trigger the instability as a function of the $1+$ current.

Figure 9(a) shows that the delay is inversely proportional to the injected current, which implies that the instability appears after the Cs concentration of the charge breeder oxygen plasma exceeds a certain threshold. This is further highlighted by Fig. 9 (b) demonstrating that, independent on the $1+$ current, the instabilities are triggered after approximately 50-60 nC positive charge has been injected. It is, however, worth noting that the injected charge does not directly translate into accumulated charge. The difference between the two presumably depends on the efficiency of the $1+$ capture process (e.g. $\Delta V$, plasma density, etc.) and ion confinement time of the charge bred $n+$ ions, which were not probed in this experiment. Nevertheless, the figure demonstrates that triggering the instabilities is not a prompt effect but instead, depending on the injected current, the instabilities can take tens or even hundreds of milliseconds to develop. The result highlights the fact that $1+$ injection affects the EEDF of the charge 

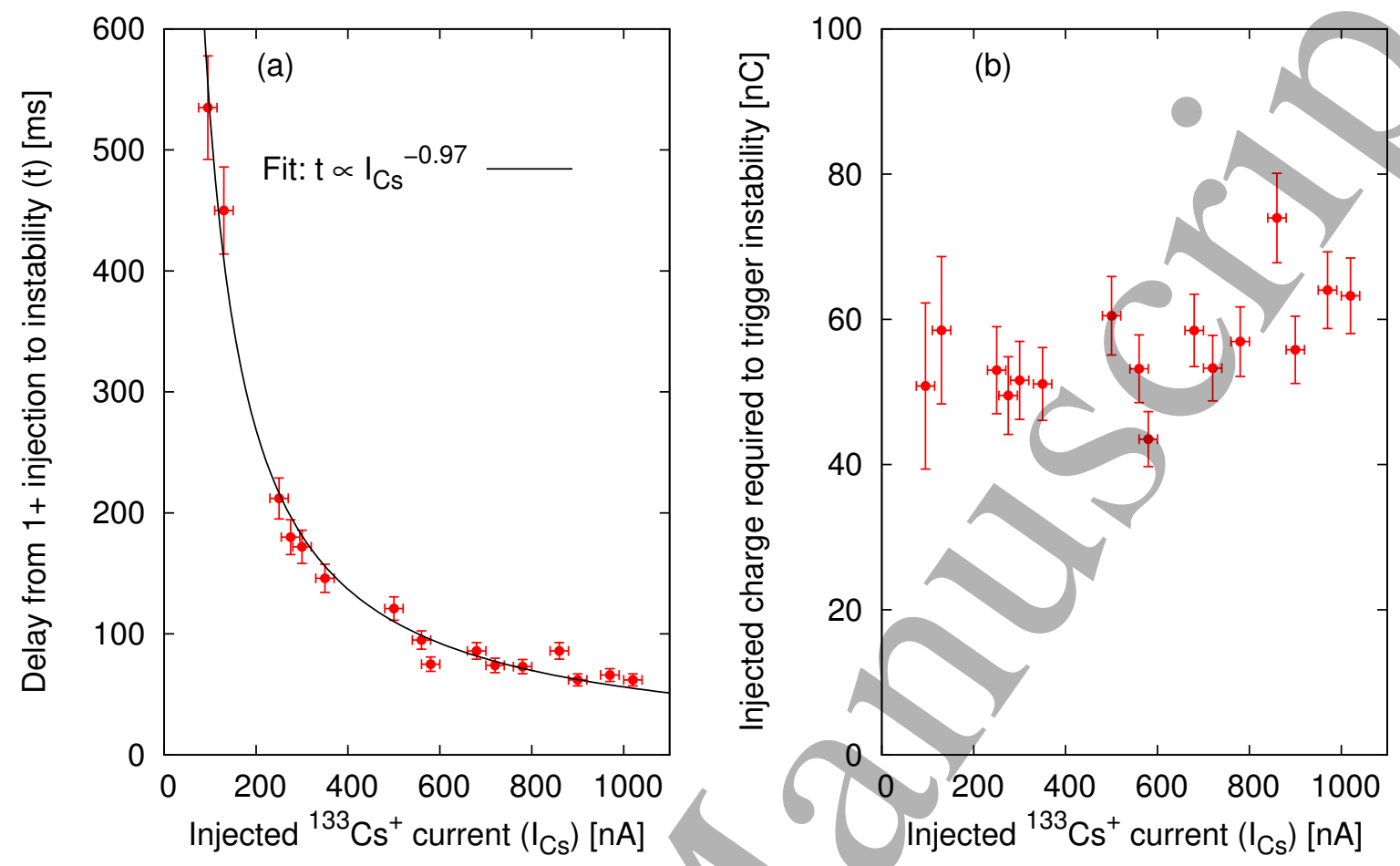

Figure 9. (a) The measured delay between the leading edge of the $\mathrm{Cs}^{+}$injection pulse and the appearance of the first instability event as a function of the injected beam current. (b) The (total) injected charge required to trigger the instability as a function of the injected beam current.

breeder plasma gradually through accumulation of the heavier species and suggests that the plasma parameters evolve drastically during the development of the Cs charge state distribution often referred as the charge breeding time. It can be expected that the actual value of the injected charge at the instability threshold depends on the $1+$ ion species and, to some degree, on the ion source settings.

Although it was demonstrated that the injection of the $1+$ ions can affect the charge breeder plasma properties and subsequently trigger instabilities, the threshold between stable and unstable plasma regimes is mainly determined by other ECRIS parameters, especially the magnetic field strength. This is demonstrated in Fig. 10 showing the $\Delta V$ curves of $\mathrm{Cs}^{26+}$ and $\mathrm{Rb}^{19+}$ measured at different settings of the magnetic field, indicated here by the corresponding $B_{\min } / B_{E C R^{-r a t i o s}}$ In the given examples the oxygen discharge was sustained by $300 \mathrm{~W}$ microwave power and the injected $1+$ currents were $300 \mathrm{nA}$ and $260 \mathrm{nA}$ for $\mathrm{Cs}$ and Rb, respectively. The data are normalized to the maximum currents obtained in stable regime at optimum $\Delta V$.

With the given source tuning the oxygen plasma remains stable at $B_{\min } / B_{E C R} \leq$ 0.85 regardless of the $1+$ injection. On the contrary at $B_{\min } / B_{E C R} \geq 0.87$ instabilities appear in the whole range of $\Delta V$ values. In the transition region the capture of the $1+$ ions at certain range of $\Delta V$ triggers instabilities as discussed above. In terms of $\Delta V$ 
Plasma instabilities of a charge breeder ECRIS
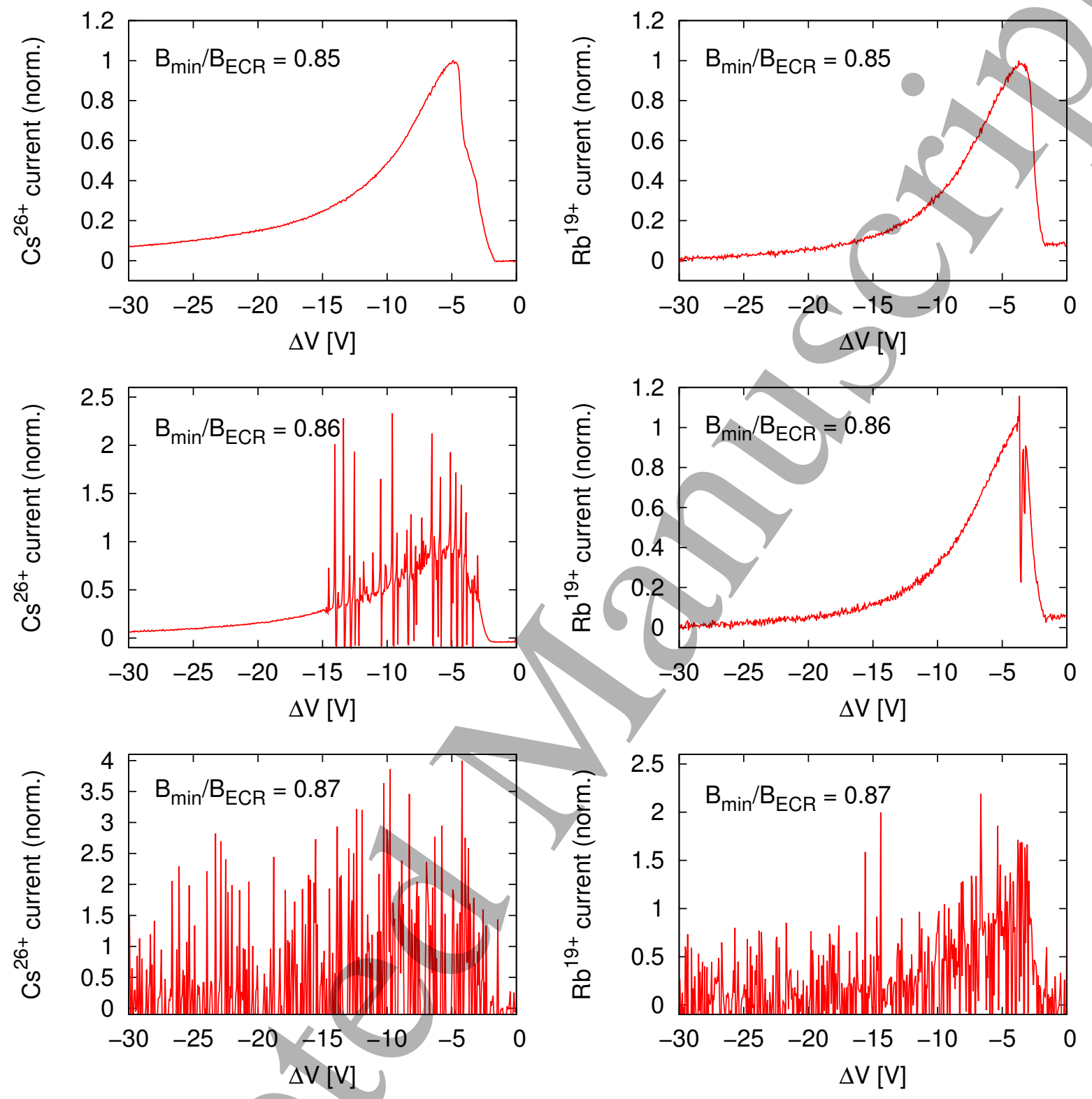

Figure 10. Examples of $\Delta V$ curves of $\mathrm{Cs}^{26+}$ and $\mathrm{Rb}^{19+}$ measured at different $B_{\text {min }} / B_{E C R}$-ratios.

values the transition region was observed to be wider for Cs than for Rb (several volts). In other words, the injection of Cs was observed to affect the charge breeder (oxygen) plasma properties more substantially in comparison to the injection of $\mathrm{Rb}$, therefore making it more prone to instabilities. This is consistent with the fact that due to the mass effect, injecting Cs affects the properties of the charge breeder plasma more than the injection of $\mathrm{Rb}$, as indicated by the change of the oxygen charge state distribution [22]. Furthermore, due to its higher atomic number Cs has a greater potential to affect the electron density and electron velocity distribution of the charge breeder plasma through electrons released by ionization in comparison to $\mathrm{Rb}$. It is worth noting that in 
Plasma instabilities of a charge breeder ECRIS

Table 1. Charge breeding efficiency of $\mathrm{Cs}^{26+} / \mathrm{Rb}^{19+}$ and bremsstrahlung power flux (normalized) as a function of the $B_{\min } / B_{E C R}$-ratio. The data were obtained with microwave power of $400 \mathrm{~W}, \Delta V$ of $-8 /-4 \mathrm{~V}$ and $1+$ beam current of $790 \mathrm{nA} / 260 \mathrm{nA}$.

\begin{tabular}{lcc}
\hline$B_{\min } / B_{E C R}$ & $\mathrm{Cs}^{26+}$ efficiency [\%] & Bremsstrahlung power flux (arb.) \\
\hline 0.74 & 4.1 & 0.32 \\
0.75 & 4.3 & 0.36 \\
0.76 & 4.7 & 0.40 \\
0.78 & 4.8 & 0.44 \\
$0.79-0.85$ & $\mathrm{~N} / \mathrm{A}$ (unstable) & $\mathrm{N} / \mathrm{A}$ (unstable) \\
0.86 & 6.1 & 1.00 \\
$>0.86$ & $\mathrm{~N} / \mathrm{A}$ (unstable) & $\mathrm{N} / \mathrm{A}$ (unstable) \\
\hline$B_{\min } / B_{E C R}$ & $\mathrm{Rb}^{19+}$ efficiency $[\%]$ & Bremsstrahlung power flux (arb.) \\
\hline 0.74 & 5.1 & 0.35 \\
0.76 & 5.0 & 0.37 \\
0.77 & 5.5 & 0.41 \\
0.78 & 5.1 & 0.47 \\
0.80 & 5.9 & 0.55 \\
0.81 & 6.4 & 0.62 \\
0.82 & 6.3 & 0.71 \\
$0.82-0.85$ & $\mathrm{~N} / \mathrm{A}($ unstable) & $\mathrm{N} / \mathrm{A}($ unstable) \\
0.86 & 5.4 & 1.00 \\
$>0.86$ & $\mathrm{~N} / \mathrm{A} /$ (unstable) & $\mathrm{N} / \mathrm{A}$ (unstable) \\
\hline
\end{tabular}

the unstable mode the currents of high charge state Cs and Rb ions momentarily exceed those obtained in the stable mode at optimum $\Delta V$. This is believed to be due to the desorption of the alkali metal atoms accumulated on the walls of the plasma chamber as discussed in detail in the following subsection.

It has been shown that kinetic instabilities limit the parameter space available for the optimization of high charge state ion production by conventional ECR ion sources [11]. This is also true for the optimization of the charge breeding efficiency as demonstrated in Table 1, listing examples of the charge breeding efficiencies of $\mathrm{Cs}^{26+}$ and $\mathrm{Rb}^{194}$ as a function of the $B_{\min } / B_{E C R^{-r a t i o, ~ t o g e t h e r ~ w i t h ~ t h e ~(n o r m a l i z e d) ~}}$ bremsstrahlung power flux measuring the energy content of the electrons lost to the walls of the plasma chamber. The data analysis demonstrates that the production of high charge state ions correlates with the energy content of the escaping electron population and reachès its maximum (breeding efficiency) at $B_{\min } / B_{E C R}$ of approximately 0.8 , which is consistent with the semiempirical scaling laws of the ECRIS magnetic field [38]. At stronger fields the instabilities limit the high charge state production. It is worth noting that the transition from the stable to unstable regime is not unambiguous but so-called "islands of stability" do exist in the studied parameter space, e.g. at $B_{\text {min }} / B_{E C R}$ of 0.86 as shown in the table. 
Another operating parameter that affects the (electron) energy content and, thus, the stability of the plasma is the microwave power. It was observed that the bremsstrahlung power flux scales linearly with the power when the magnetic field strength was kept constant. It has been shown previously that the electron density of the charge breeder plasma is well below the critical (cut-off) density and increases almost linearly with increasing microwave power [22]. Therefore, the increase of bremsstrahlung power flux can be attributed to increasing electron density. The combined effect of the magnetic field strength and the microwave power on the plasma stability is depicted in Fig. 11. The figure shows a two-dimensional parametric $\left(B_{\min } / B_{E C R}\right.$, microwave power) stability map with and without $\mathrm{Cs}^{+}$or $\mathrm{Rb}^{+}$injection at several $1+$ currents.
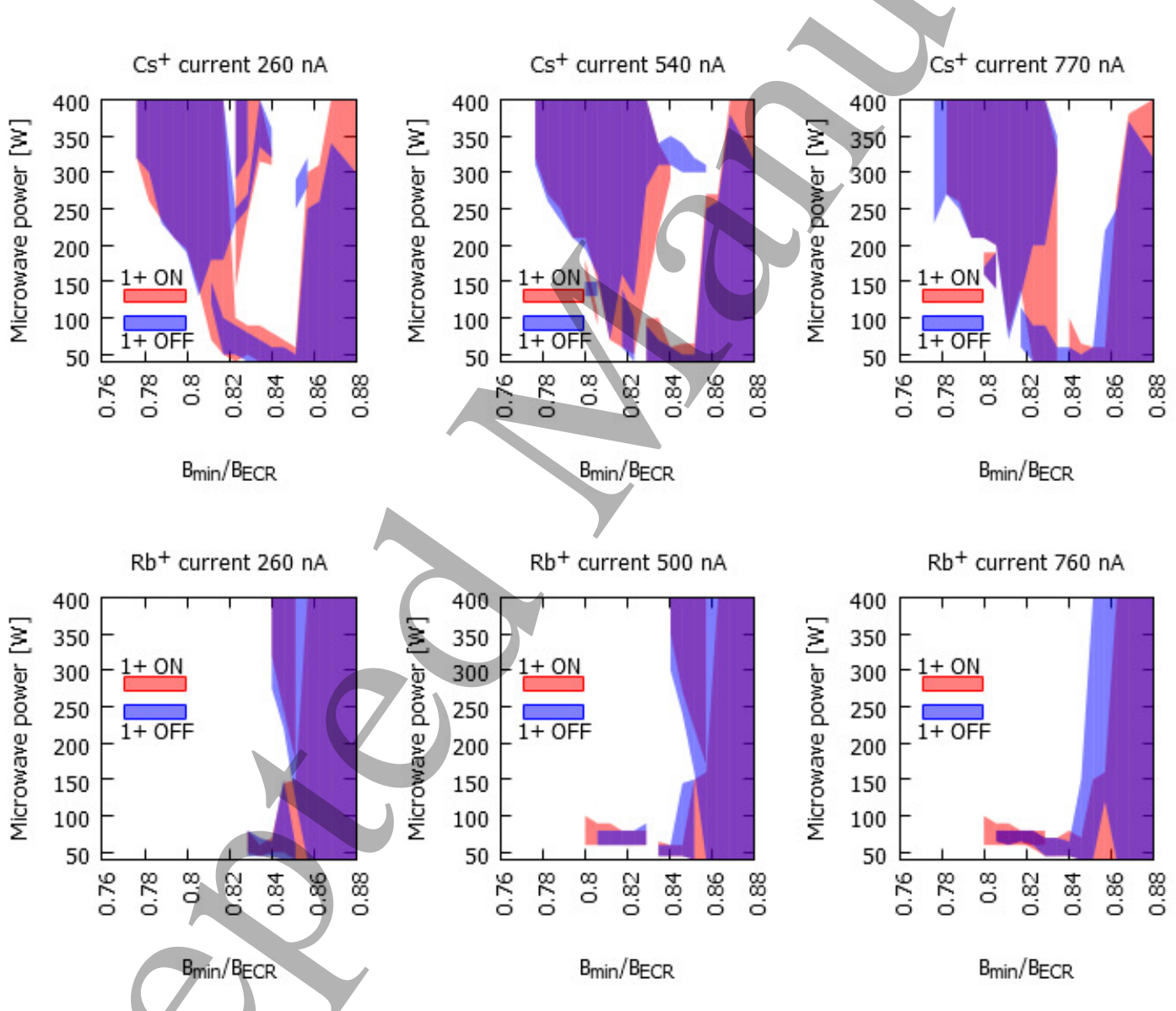

Figure 11. The combined effect of the magnetic field strength and the microwave power on the plasma stability with and without $\mathrm{Cs}^{+}$or $\mathrm{Rb}^{+}$injection at several $1+$ currents. The white (background) color refers to parameter settings corresponding to stable plasma. Unstable regimes are denoted with transparent red (1+ injection $\mathrm{ON})$ and transparent blue $(1+$ injection $\mathrm{OFF})$ or a combination of the two.

The data in Fig. 11 permits to make several observations: (i) The transition from stable to unstable regime is affected most by the magnetic field strength while the microwave power plays a less significant role. (ii) So-called "islands of stability" can be observed in the 2D parameter space as clearly seen in the Cs data sweep. (iii) Injection 
and capture of the $\mathrm{Cs}^{+}$beam causes the unstable regimes of the parameter space to expand slightly (red color). However, at certain parameter combinations, the capture of the $1+$ beam can also stabilize the oxygen discharge (blue color). Altogether, the effect of the $1+$ injection is less significant in comparison to the magnetic field strength as demonstrated by the overlap of the colors in Fig.11 indicating the regions of the parameter space where the plasma was observed to be always unstable regardless of the $1+$ injection. The implications of this observation are discussed further in the following Section. (iv) The effect of the $1+$ capture on the plasma stability is pronounced for Cs in comparison to Rb. This is most likely due to the mass effect discussed above.

\subsection{The effect of plasma instabilities on impurities of the extracted ion beam}

The presence of impurities in the extracted ion beam is often considered as the major drawback of ECRIS charge breeders in comparison to their EBIS counterparts [5]. The root cause for the existence of the impurities is the interaction between the energetic plasma particles and the walls of the plasma confinement yessel. In ECRIS the plasma is in direct contact with the walls of the plasma chamber unlike in EBIS. The charged particle losses are concentrated primarily to six plasma flutes reflecting the topology of the sextupole cusp field. The plasma-wall interaction results to adsorption and sputtering of impurities.

The plasma-wall interaction is enhanced in the unstable ECRIS plasma regime due to the loss of ion confinement associated with each onset of the instability. The effect of the instabilities on the extracted currents of oxygen ion beams is demonstrated in Fig. 12 showing the temporally averaged charge state distribution in stable $\left(B_{\min } / B_{E C R}=0.80\right)$ and unstable $\left(B_{\min } / B_{E C R}=0.85\right)$ operating regimes with $420 \mathrm{~W}$ microwave power. The absolute values of the intensities are low since the data was recorded using the aforementioned pair of $0.2 \mathrm{~mm}$ slits in front of the Faraday cup. The numbers associated with each charge state refer to the ratio of currents obtained in unstable and stable mode, respectively. It is evident that due to reduced ion confinement time the instabilities limit the production of the high charge states while the temporally averaged currents of low charge states $(\leq 3+)$ are hardly affected.

The instabilities expel a significant fraction of the electrons [39], which results to a significant increase of the plasma potential compensating the electron and ion losses. It has been reported that the plasma potential reaches values of $\geq 1 \mathrm{kV}$ during the instability-induced transient lasting some tens of microseconds [18] while the corresponding value under stable operation of the charge breeder is on the order of $10 \mathrm{~V}$ [31]. The considerable (up to two orders of magnitude) increase of the plasma potential results to enhanced sputtering of the plasma chamber wall material and residues desorbing from the surface. For oxygen charge states $\leq 3$, which contribute most to the ion density, the difference in sputter yield of iron, being the most abundant element in the stainless steel material of the PHOENIX CB-ECRIS plasma chamber, at enêrgies corresponding to a plasma potential of $10 \mathrm{~V}$ and $1 \mathrm{kV}$ is on the order of $10^{3}$ [40]. 


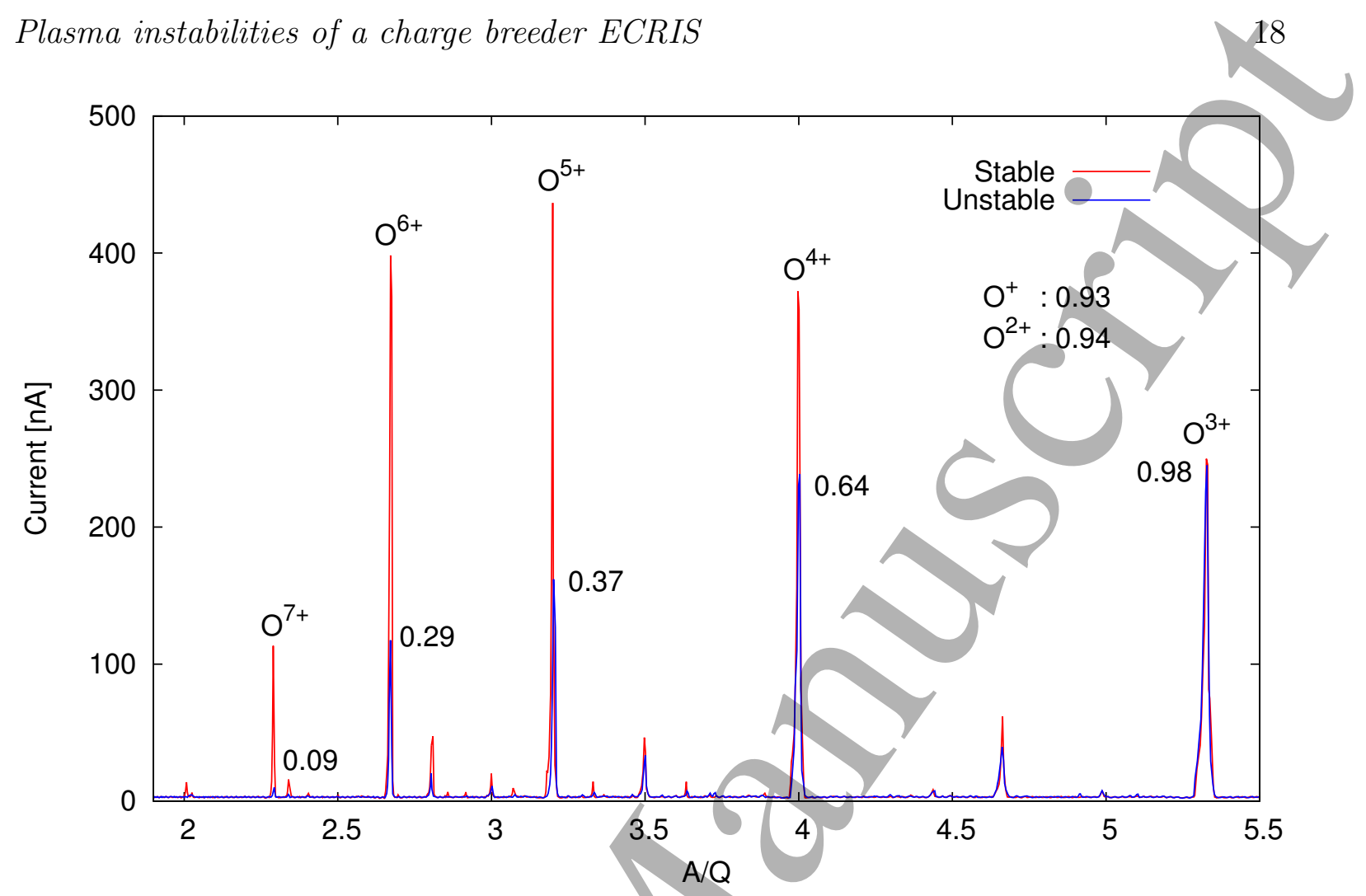

Figure 12. An example of the charge state distribution of the oxygen plasma in stable and unstable regimes. The numbers associated with each charge state refer to the ratio of currents obtained in unstable and stable mode, respectively.

Thus, despite of the short duration of the plasma potential transient it can be expected that the kinetic instabilities affect the level of impurities in the extracted beam. This is demonstrated in Fig. 13 showing the temporally averaged $m / q$-resolved spectrum at high sensitivity / low current in two ranges of $A / Q$ (mass/charge in atomic mass units) values. The ion source settings correspond to those used for obtaining the data for Fig. 12 above. The figure clearly demonstrates that in the unstable regime the extracted currents of impurities are significantly higher in comparison to stable operation. It is worth noting that the average intensities of gaseous impurities, e.g. argon, present due to an insignificant air leak are not pronounced in the unstable operation mode, which implies that the amplification of the impurity peaks is due to plasma wall interaction. The given $A / Q$-ranges were chosen for illustration because at lower $A / Q$ the CSD was observed to be less affected by the instabilities. This is because the high charge state ion currents are suppressed in the unstable regime.

Table 2 lists all the $A / Q$-values at which the recorded beam currents in unstable regime were observed to exceed those obtained in the stable regime by a factor of two at least. The most probable candidates for the impurity ion species are identified and the corresponding ratios of the currents in unstable $\left(I_{U}\right)$ and stable $\left(I_{S}\right)$ regimes are listed in the table as well. The appearance of the prominent impurity species (Fe, $\mathrm{Cr}$, Mo, Ni, Al, Zn, Cu, Mn) can be explained by sputtering of the plasma chamber made 
Plasma instabilities of a charge breeder ECRIS
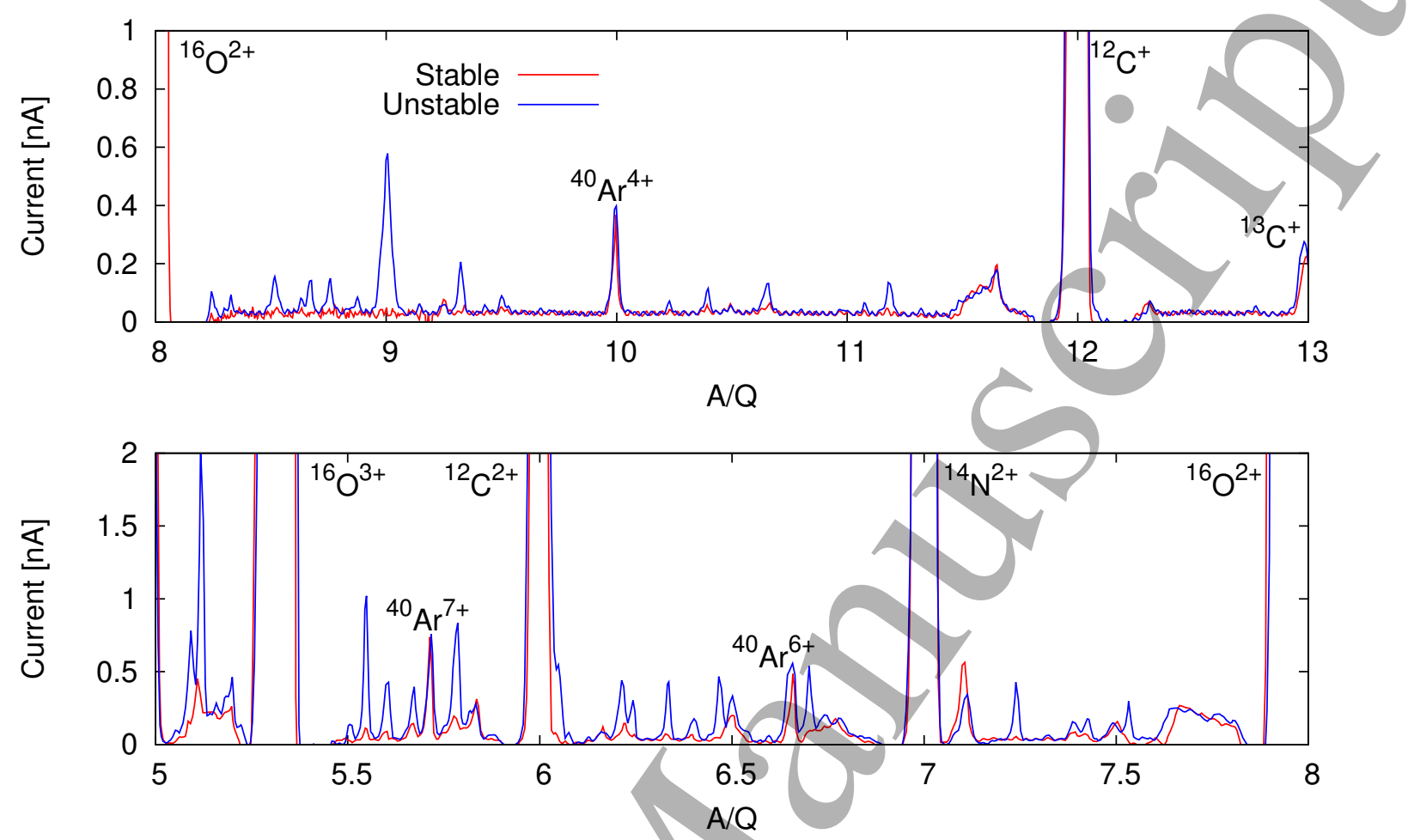

Figure 13. Temporally averaged $\mathrm{m} / \mathrm{q}-$-resolved spectra at high sensitivity/low current in the ranges of 8-13 (top) and 5-8 (bottom).Carbon, nitrogen, oxygen and argon ions are indicated for convenience.

of 316L stainless steel (Fe, <0.03\% C, 16-18.5\% Cr, 10-14\% Ni, 2-3\% Mo) and plasma electrode / RF blocker electrode in the injection [41] made of AU4G aluminum ( $\mathrm{Al}$, $3.5-4.5 \% \mathrm{Cu}, 0.4-1 \% \mathrm{Mn}, 0.25 \% \mathrm{Zn}$ ), which are in direct contact with the plasma. It is worth noting that different charge states of ${ }^{133} \mathrm{Cs}$ and ${ }^{85} \mathrm{Rb}$ also appear in the unstable mode. This is probably due to release of the alkali metals accumulated on the walls of the plasma chamber over several days of the charge breeding experiments described above. The relative increase of each element/isotope in the $m / q$-analyzed spectrum does not directly correspond to the composition of the metal alloy nor the natural isotopic ratio since the extracted current depends on the periodicity of the plasma instabilities and scanning speed of the dipole magnet current ramp. In other words, the spectrum can be used only for identifying impurities, not quantifying their relative abundancies. The complete list of the impurity species is presented in order to highlight that the spectrum of the background contamination of the extracted ion beams depends on the choice of the plasma facing materials and is therefore specific to each charge breeder ion source.

\section{Conclusions}

The presented study has demonstrated the significance of electron cyclotron instabilities on the operation of a charge breeder ECRIS. The main conclusions of the work are: 
Plasma instabilities of a charge breeder ECRIS

Table 2. List of (most probable) impurities affected by the instabilities. $I_{U} / I_{S}$ refers to the ratio of beam currents recorded in unstable $(\mathrm{U})$ and stable $(\mathrm{S})$ operation regimes.

\begin{tabular}{|c|c|c|c|c|c|}
\hline$A / Q$ & $\operatorname{Ion}(\mathrm{s})$ & $I_{U} / I_{S}$ & $A / Q$ & $\operatorname{Ion}(\mathrm{s})$ & $I_{U} / I_{S}$ \\
\hline $26.99-27.02$ & ${ }^{54} \mathrm{Fe}^{2+},{ }^{27} \mathrm{Al}^{1+}$ & 2.1 & $6.23-6.25$ & $\mathrm{r}^{8+},{ }^{100} \mathrm{M}$ & 14 \\
\hline $25.98-26.02$ & ${ }^{52} \mathrm{Cr}^{2+}$ & 2.5 & $6.21-6.23$ & $e^{9+}$ & .1 \\
\hline $18.58-18.62$ & ${ }^{56} \mathrm{Fe}^{3+}$ & 7.1 & $5.87-5.89$ & OR & 3.6 \\
\hline $11.86-11.11$ & ${ }^{100} \mathrm{Mo}^{9+}$ & 2.5 & $5.77-5.79$ & & 4.8 \\
\hline $11.19-11.21$ & ${ }^{56} \mathrm{Fe}^{5+}$ & 6.2 & $5.67-5.68$ & & 3.1 \\
\hline $10.66-10.68$ & ${ }^{96} \mathrm{Mo}^{9+}$ & 3.4 & $5.59-5.61$ & & 6.8 \\
\hline $10.40-10.45$ & ${ }^{94} \mathrm{Mo}^{9+},{ }^{52} \mathrm{Cr}^{5+}$ & 4.1 & $5.52-5.55$ & $\mathrm{~s}^{24+},{ }^{61} \mathrm{Ni}^{11+}$ & 11 \\
\hline $10.22-10.25$ & ${ }^{92} \mathrm{Mo}^{9+}$ & 3.2 & $5.49-5.50$ & ${ }^{66} \mathrm{Zn}^{12+}$ & 2.4 \\
\hline $9.50-9.52$ & ${ }^{57} \mathrm{Fe}^{6+},{ }^{95} \mathrm{Mo}^{10+}$ & 4.2 & $5.19-5$ & ${ }^{52} \mathrm{Cr}^{10+}$ & 5.3 \\
\hline $9.33-9.34$ & ${ }^{56} \mathrm{Fe}^{6+}$ & 6.0 & $5.12-5.13$ & ${ }^{133} \mathrm{Cs}^{26+}$ & 5.4 \\
\hline $9.17-9.20$ & ${ }^{92} \mathrm{Mo}^{10+}$ & 3.0 & 5.09 & ${ }^{56} \mathrm{Fe}^{11+}$ & 6.8 \\
\hline $8.99-9.01$ & ${ }^{54} \mathrm{Fe}^{6+},{ }^{27} \mathrm{Al}^{3+}$ & 55 & $4.93-4.95$ & ${ }^{133} \mathrm{Cs}^{27+}$ & 4.4 \\
\hline $8.85-8.90$ & ${ }^{98} \mathrm{Mo}^{11+},{ }^{62} \mathrm{Ni}^{7+},{ }^{133} \mathrm{Cs}^{15+}$ & 5.5 & $4.86-4.88$ & ${ }^{68} \mathrm{Zn}^{14+}$ & 5.7 \\
\hline $8.72-8.76$ & ${ }^{96} \mathrm{Mo}^{11+},{ }^{61} \mathrm{Ni}^{7+}$ & 7.1 & $4.84-4.86$ & ${ }^{58} \mathrm{Ni}^{12+}$ & 4.2 \\
\hline $8.66-8.68$ & ${ }^{52} \mathrm{Cr}^{6+}$ & & $4.75-4.76$ & ${ }^{133} \mathrm{Cs}^{28+}$ & 4.8 \\
\hline $8.62-8.64$ & ${ }^{95} \mathrm{Mo}^{11+}$ & & 3 & ${ }^{52} \mathrm{Cr}^{11+}$ & 6.0 \\
\hline $8.50-8.52$ & ${ }^{17} \mathrm{O}^{2+}$ & 7. & $4.59-4.60$ & ${ }^{133} \mathrm{Cs}^{29+}$ & 3.4 \\
\hline $8.32-8.34$ & $6+$ & & $4.57-4.59$ & ${ }^{55} \mathrm{Mn}^{12+}$ & 4.7 \\
\hline $8.26-8.29$ & & 3 & $4.53-4.55$ & ${ }^{50} \mathrm{Cr}^{11+},{ }^{68} \mathrm{Zn}^{15+}$ & 8.7 \\
\hline $8.23-8.25$ & & 7.4 & $4.48-4.50$ & ${ }^{27} \mathrm{Al}^{6+},{ }^{54} \mathrm{Fe}^{12+}$ & 2.6 \\
\hline $7.54-7.57$ & $13+$ & 13 & $4.40-4.42$ & ${ }^{53} \mathrm{Cr}^{12+}$ & 8.7 \\
\hline $7.43-7.45$ & $7+$ & 3.7 & $4.33-4.35$ & ${ }^{52} \mathrm{Cr}^{12+}$ & 3.9 \\
\hline $7.38-7.40$ & 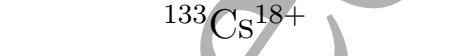 & 2.5 & $4.31-4.33$ & ${ }^{56} \mathrm{Fe}^{13+}$ & 7.3 \\
\hline $7.23-7.26$ & & 20 & $4.27-4.29$ & ${ }^{60} \mathrm{Ni}^{14+}$ & 16 \\
\hline $6.74-6.76$ & ${ }^{54} \mathrm{Fe}^{8+}$ & 2.2 & $4.25-4.27$ & ${ }^{64} \mathrm{Zn}^{15+}$ & 3.0 \\
\hline $6.71-6.73$ & $\mathrm{Mo}^{14+}$ & 8.5 & $4.15-4.17$ & ${ }^{50} \mathrm{Cr}^{12+},{ }^{133} \mathrm{Cs}^{32+}$ & 9.5 \\
\hline $6.60-6.63$ & $\mathrm{Cr}^{8+}$ & 3.1 & $3.79-3.80$ & ${ }^{57} \mathrm{Fe}^{15+},{ }^{53} \mathrm{Cr}^{14+}$ & 3.0 \\
\hline $6.50-6.52$ & & 2.1 & $3.77-3.79$ & ${ }^{68} \mathrm{Zn}^{18+}$ & 5.6 \\
\hline $6.49-6.51$ & ${ }^{52} \mathrm{Cr}^{8+}$ & 22 & $3.73-3.75$ & ${ }^{56} \mathrm{Fe}^{15+}$ & 5.7 \\
\hline $6.39-6.41$ & $\mathrm{Zn}^{10+},{ }^{96} \mathrm{Mo}^{15+}$ & 6.0 & $3.71-3.73$ & ${ }^{52} \mathrm{Cr}^{14+}$ & 2.4 \\
\hline $6.32-6.34$ & ${ }^{57} \mathrm{Fe}^{9+}$ & 13 & $3.30-3.32$ & ${ }^{56} \mathrm{Fe}^{15+}$ & 3.3 \\
\hline
\end{tabular}


(i) The experiments have confirmed that the kinetic instabilities, observed and studied earlier with the conventional JYFL 14 GHz ECRIS $[11,12,30]$ possess characteristic features common to minimum-B ECR ion sources. In particular these include microwave emission in sequential bursts having a falling tone of frequencies, severe losses of relativistic electrons causing sharp bursts of bremsstrahlung emission and periodic loss of ion confinement leading to significant increase of the plasma potential and drop of extracted currents of high charge state ions thus limiting the parameter space available for the optimization of the ion source performance. The fact that in charge breeder ECRIS the instabilities appear at higher $B_{\min } / B_{E C R^{-}}$ ratio and lower (on-axis) field gradient in comparison to conventional ECRIS [11] calls for further experiments to study whether the instability threshold depends on the absolute field strength (electron confinement) instead of magnetic field gradient (electron heating).

(ii) It was demonstrated that under certain conditions, i.e. microwave power and magnetic field configuration, the injection of the $1+$ beam perturbs the charge breeder plasma sufficiently to trigger (or sometimes suppress) kinetic instabilities in oxygen discharge. Although the effect of the $1+$ injection is less significant in comparison to the magnetic field configuration for example, the appearance of the instabilities can limit the breeding efficiency of high charge state ions.

(iii) It was observed that there is a significant delay between the leading edge of the $1+$ pulse and the first appearance of the instabilities. The length of the delay was observed to depend on the injected $1+$ current. This excludes a prompt beam-plasma interaction, discussed in Refs. [36, 37], being an explanation for the observed instabilities. On the contrary, the instabilities are due to accumulation and subsequent interaction of the injected ions with the plasma electrons (ionization) and buffer gas ions (ion-ion collisions) resulting to increased growth rate and/or decreased damping rate of the kinetic instabilities.

(iv) It was observed that the transition from stable to unstable regime of the oxygen discharge is more sensitive to the injection of ${ }^{133} \mathrm{Cs}$ in comparison to ${ }^{85} \mathrm{Rb}$. This implies that the level of the perturbation depends on the incident ion mass and subsequent degree of ionization. The observation has far-reaching consequences. It can be argued that the (delayed) appearance of instabilities is due to the variation of the electron energy distribution in a time-scale similar to the reported breeding times (90\% rise times) of the high charge states of the injected ions [29]. Since the method of measuring the breeding time, i.e. pulsing the $1+$ injection, clearly affects the charge state distribution of the buffer gas discharge and its electron energy distribution as demonstrated here, the actual breeding times in continuous operation can differ significantly from those obtained by studying the injection transient.

(v) The effect of the instabilities on the level of impurities released from the plasmafacing surfaces in the extracted ion beam was demonstrated. The impurities are 
released due to plasma-wall interaction driven by the fluctuation of the plasma potential [18]. Operating the charge breeder ECRISs near the threshold between stable and unstable discharge regimes should be avoided in order to reduce the amount of impurities. However, optimizing the breeding efficiency often requires high microwave power and strong magnetic field, which makes the plasma prone to kinetic instabilities. Such conflict between optimization of the charge breeding efficiency and preserving the purity of the extracted beams calls for installation of appropriate diagnostics, capable of detecting the instabilities, on charge breeder ECRISs. The instability-induced sputtering can explain the erosion of the plasma chamber observed in afterglow operation of the GTS ion source with argon at CERN [42].

\section{Acknowledgements}

The project has been funded by the Université Grenoble-Alpes EMERGENCE programme.

\section{References}

[1] R. Geller, C. Tamburella and J.L. Belmont, Rev. Sci. Instrum. 67, (1996), pp. 1281-1285.

[2] Y. Blumenfeld, T. Nilsson and P. Van Duppen, Phys. Scr., T152, (2013), 014023.

[3] R. Geller, T. Lamy and P. Sortais, Rev. Sci. Instrum. 77, 03B107 (2006).

[4] R.E. Marrs and D.R. Slaughter, AIP Conf. Proc. 475, (1999), p. 322.

[5] R. Vondrasek, Nucl. Instr. Meth. Phys. Res. B 376 (2016) 16-23.

[6] F. Ames, M. Marchetto, A. Mjøs and A.C. Morton, Rev. Sci. Instrum. 87(2), 02B501, (2016).

[7] L. Maunoury, P. Delahaye, M. Dubois, J. Angot, P. Sole, O. Bajeat, C. Barton, R. Frigot, A. Jeanne, P. Jardin, O. Kamalou, P. Lecomte, B. Osmond, G. Peschard, T. Lamy, and A. Savalleless, Rev. Sci. Instrum. 87(2), 02B508, (2016).

[8] S. Gales, AIP Conf. Proc. 1355, 36 (2011).

[9] G. Bisoffi et al., Nucl. Instr. Meth. Phys. Res. B 376 (2016) 402-407.

[10] A. Herlert and Y. Kadi, J. Phys. Conf. Ser. 312, 052010, (2011).

[11] O. Tarvainen, J. Laulainen, J. Komppula, R. Kronholm, T. Kalvas, H. Koivisto, I. Izotov, D. Mansfeld and V. Skalyga, Review of Scientific Instruments 86, 023301 (2015).

[12] O. Tarvainen, I. Izotoy, D. Mansfeld, V. Skalyga, S. Golubev, T. Kalvas, H. Koivisto, J. Komppula, R. Kronholm and J. Laulainen, Plasma Sources Sci. Technol. 23, 2 (2014).

[13] C. Barue, M. Lamoreux, P. Briand, A. Girard and G. Melin, J. Appl. Phys. 76, 5, (1994).

[14] G. Douysset, H. Khodja, A. Girard and J.P. Briand, Phys. Rev. E 61, 3, (2000).

[15] H. Tawara and T. Kato, Atomic Data and Nuclear Data Tables 36, 2, (1987).

[16] V.P. Pastoukhov, Rev. Plasma Phys. 13, (1987), p. 203.

[17] T. Rognlien and T. Cutler, Nucl. Fusion 20, (1980), p. 1003.

[18] O. Tarvainen, T. Kalvas, H. Koivisto, J. Komppula, R. Kronholm, J. Laulainen, I. Izotov, D. Mansfeld, V. Skalyga, V. Toivanen and G. Machicoane, Rev. Sci. Instrum. 87, 02 A703 (2016).

[19] T. Lamy, J. L. Bouly, J. C. Curdy, R. Geller, A. Lacoste, P. Sole, P. Sortais, T. Thuillier, J.L. Vieux-Rochaz, K. Jayamanna, M. Olivo, P. Schmor, and D. Yuan, Rev. Sci. Instrum. 73, 717 (2002).

[20] T. Lamy, J. Angot and C. Fourel, WE-06 in Proc. HIAT2009, Venice, Italy, (2009), JACoW.org [21] J.L. Delcroix and A. Bers, Physique des Plasmas, CNRS, New York, (1994), Vol. 2, p.357. 
[22] O. Tarvainen, T. Lamy, J. Angot, T. Thuillier, P. Delahaye, L. Maunoury, J. Choinski, L. Standylo, A. Galatà, G. Patti and H. Koivisto, Plasma Sources Sci. Technol.24, 3 (2015).

[23] T. Lamy, J. Angot, T. Thuillier, P. Delahaye, L. Maunoury, J. Choinski, L. Standylo, A. Galatà, G. Patti, H. Koivisto and O. Tarvainen, WEOBMH01 in Proc. ECRIS2014, Nizhny Novgorod, Russia, (2014), JACoW.org

[24] J. Angot, T. Lamy, M. Marie-Jeanne, P. Sortais and T. Thuillier, THYO03 in Proc. ECRIS2012, Sydney, Australia, (2012), JACoW.org

[25] T. Lamy, J.-L. Bouly, J.-C. Curdy, P. Sole, P. Sortais, T. Thuillier, J.-L. Vieux-Rochaz, and D. Voulot, Rev. Sci. Instrum. 75, 1624 (2004).

[26] J. Orpana, O. Tarvainen, T. Kalvas, H. Koivisto, R. Kronholm, J. Laulainen, I. Izotov, D. Mansfeld and V. Skalyga, to be published in Proc. ECRIS2016, Busan, Korea, (2016), JACoW.org

[27] http://www.esrf.eu/Accelerators/Groups/InsertionDevices/Software/Radia/

[28] J. Benitez, C. Lyneis, L. Phair, D. Todd and D. Xie, IEEE Trans. Plasma Sci., Vol. 45, No. 7, (2017).

[29] P. Delahaye, A. Galatà, J. Angot, J. F. Cam, E. Traykoy, G. Ban, L. Celona, J. Choinski, P. Gmaj, P. Jardin, H. Koivisto, V. Kolhinen, T. Lamy, L. Maunoury, G. Patti, T. Thuillier, O. Tarvainen, R. Vondrasek, and F. Wenander, Rev. Sci. Instrum. 87, 02B510 (2016).

[30] I. Izotov, O. Tarvainen, D. Mansfeld, V. Skalyga, H. Koivisto, T. Kalvas, J. Komppula, R. Kronholm and J. Laulainen, Plasma Sources Sci. Technol. 24, 4 (2015).

[31] T. Lamy, J. Angot, M. Marie-Jeanne, J. Medard, P. Sortais, T. Thuillier, A. Galatà, H. Koivisto and O. Tarvainen, WECOBK03 in Proc. ECRIS2010, Grenoble, France, (2010), JACoW.org

[32] A.G. Drentje, Nucl. Instr. Meth. Phys. Res. B 9 (1985), p. 526.

[33] A.G. Drentje, A. Girard, D. Hitz and G. Melin, Rev/Sci. Instrum. 71, (2000), p. 623.

[34] V. Mironov, S. Bogomolov, A. Bondarchenko, A. Efremov, and V. Loginov, Phys. Rev. Accel. Beams 20, 013402 (2017).

[35] O. Tarvainen, H. Koivisto, A. Galatà, J. Angot, T. Lamy, T. Thuillier, P. Delahaye, L. Maunoury, D. Mascali and L. Neri, Phys. Rev. Accel. Beams 19, 053402, (2016).

[36] A. Galatà, D. Mascali, L. Neri and L. Celona, Plasma Sources Sci. Technol. 25, 4 (2016).

[37] J. Neufeld and H. Wright, Phys. Rev. Lett. 129, 4 (1963).

[38] D. Hitz, A. Girard, G. Melin, S. Gammino, G. Ciavola and L. Celona, Rev. Sci. Instrum. 73, (2002), p. 509.

[39] S.A. Hokin, R.S. Post, and D.L. Smatlak, Phys. Fluids B: Plasma Physics 1, 862 (1989).

[40] https://www.iap.tuwien.ac.at/www/surface/sputteryield

[41] T. Lamy, J. Angot, P. Sortais, T. Thuillier and A. Galatà, Rev. Sci. Instrum. 83, 02A909 (2012).

[42] D. Küchler, J. Ferreira Somoza, A. Michet and V. Toivanen, to be published in Proc. ECRIS2016, Busan, Korea, (2016), JACoW.org 